\title{
EQUATIONALLY COMPLETE DISCRIMINATOR VARIETIES OF GROUPOIDS ${ }^{1}$
}

\author{
ROBERT W. QUACKENBUSH
}

\begin{abstract}
J. Kalicki proved that there are continuum many equationally complete varieties of groupoids. In this note we give a constructive proof of this by defining a countable partial groupoid which has continuum many completions such that each completion generates an equationally complete variety, and no two distinct completions generate the same variety. Moreover, the variety generated by all the completions is a discriminator variety, and every nontrivial groupoid in this variety is cancellative but not a quasigroup; this answers a question of R. Padmanabhan. A. D. Bol'bot proved a similar result for loops, but his computations are more difficult since his varieties are not discriminator varieties.
\end{abstract}

Kalicki proved his theorem [5] by constructing continuum many consistent equational theories such that the join of any two of them is inconsistent. By the compactness theorem for equational logic, each has an extension to an equationally complete theory. Since the original theories were pairwise inconsistent, the equationally complete extensions are pairwise distinct. Thus, there are continuum many equationally complete equational theories of groupoids.

This beautiful application of the compactness theorem has the nagging drawback that is common to all compactness results: we know that all these equationally complete theories exist but we know neither an equational base nor a nontrivial model of any of them. Indeed, since an equationally complete variety is generated by each of its nontrivial members, we would know the variety if we knew just one nontrivial member. Bol'bot has rectified this in [1] where he constructs continuum many countable totally symmetric quasigroups which generate pairwise distinct equationally complete varieties. His approach is similar to mine with the exception that he did not have the theory of discriminator varieties available; consequently, his constructions are much more difficult. A nice discussion of these and related constructions can be found in the lecture notes [7] of D. Pigozzi.

In this note I will show how to construct continuum many countable groupoids such that each generates an equationally complete variety, the varieties being pairwise distinct. The basic idea is taken from the study of Sheffer functions on finite sets. In addition, these varieties consist of cancellative groupoids which are not

Received by the editors November 30, 1982 and, in revised form, May 10, 1983.

1980 Mathematics Subject Classification. Primary 08B05, 08B10.

Key words and phrases. Groupoid, variety, equational completeness, discriminator variety, quasigroup.

${ }^{1}$ Research for this paper was supported by a grant from the NSERC of Canada. 
quasigroups. R. Padmanabhan had asked whether it was possible for a variety of groupoids to be cancellative but not be quasigroups.

Definition. Let $A$ be a finite set and $f: A^{2} \rightarrow A$. We say that $f$ is Sheffer if every function on $A$ (of every finite arity) is a term function in $f$ (equivalently, if the groupoid $\underline{A}=\langle A ; f\rangle$ is primal). If $f$ is a partial function (defined on some subset of $A^{2}$ ), we say that $f$ is a partial Sheffer function if every extension of $f$ to a function on $A$ is a Sheffer function.

Our interest in Sheffer functions comes from the fact that a primal groupoid generates an equationally complete variety. (A remarkable theorem of V. L. Murskii [6] implies that almost every finite groupoid generates an equationally complete variety.) But only countably many equationally complete varieties are generated by a finite groupoid. On the other hand, there are no Sheffer functions on a countable set since there are only countably many term functions on a countable groupoid but uncountably many functions on a countable set. However, there are certain nice algebraic properties which a primal groupoid has which imply that the variety it generates is equationally complete. If a countable groupoid has these properties, then it too generates an equationally complete variety. As with partial Sheffer functions, we can define a partial groupoid on the integers $Z$ such that every extension to a groupoid on $Z$ has the desired algebraic properties, and distinct algebras generate distinct varieties.

DEFINITION. The ternary discriminator $t(x, y, z)$ is defined on any set $A$ by: for all $a, b, c \in A, t(a, a, b)=b$ and if $a \neq b$, then $t(a, b, c)=a$. A variety for which there is a term $p(x, y, z)$, such that $p$ is the ternary discriminator on every subdirectly irreducible algebra in the variety, is called a discriminator variety.

Theorem 1 (A. Foster ANd A. Pixley [2]). A finite algebra $\underline{A}=\langle A ; F\rangle$ is primal if and only if $t(x, y, z)$ is a term function of $\underline{A}$ and every $a \in A$ is the value of $a$ constant term function of $\underline{A}$.

Lemma 2. Let $\underline{A}=\langle A ; \circ\rangle$ be a countable groupoid such that $t(x, y, z)$ is a term function of $\underline{A}$ and every $a \in A$ is the value of a constant term function of $\underline{A}$. Then $\operatorname{HSP}(\underline{A})$, the variety generated by $\underline{A}$, is an equationally complete discriminator variety. Moreover, the variety generated by $\underline{A}$ coincides with the quasivariety generated by $\underline{A}$.

Proof. Since $t(x, y, z)$ is a term function, $\operatorname{HSP}(\underline{A})$ is congruence distributive. By Jonsson's Theorem [4], $\operatorname{HSP}(\underline{A})=P_{s} \operatorname{HSP}_{u}(\underline{A})$. Now, $t(x, y, z)$ is a term function on each member of $\operatorname{SP}_{u}(\underline{A})$; hence, each member of $\operatorname{SP}_{u}(\underline{A})$ is simple so $\operatorname{HSP}_{u}(\underline{A})=$ $\mathrm{SP}_{u}(\underline{A})$. Thus, $\operatorname{HSP}(\underline{A})$ is a discriminator variety and coincides with the quasivariety generated by $\underline{A}$. Since each member of $A$ is the value of a constant term function, each member of $\operatorname{SP}_{u}(\underline{A})$ contains $\underline{A}$ as a subalgebra. Thus each nontrivial algebra in $\operatorname{HSP}(\underline{A})$ contains $\underline{A}$ as a subalgebra. This means $\operatorname{HSP}(\underline{A})$ is equationally complete.

Definition. A partial groupoid $\langle A ; \circ\rangle$ is cancellative if for all $a, b, c \in A$, $a \circ b=a \circ c$ implies $b=c$ and $b \circ a=c \circ a$ implies $b=c$. A cancellative groupoid is a quasigroup if for all $a, b \in A$ there exist $x, y \in A$ such that $a \circ x=b$ and $y \circ a=b$. 
LEMMA 3. Let $\mathbf{V}$ be a variety of groupoids such that all subdirectly irreducible groupoids in $\mathbf{V}$ are cancellative. Then every groupoid in $\mathbf{V}$ is cancellative.

Proof. Cancellativity is preserved by subdirect products.

Thus, our task is to define a cancellative partial groupoid on $Z$ such that $t(x, y, z)$ is a term function and, for each $n \in Z$, there is a term function $c_{n}(x)$ constantly equal to $n$. If we have done this, then any two distinct extensions will yield nonisomorphic groupoids. By the proof of Lemma 2, this means they will generate distinct varieties. Moreover, if an extension is cancellative, then all groupoids in the variety it generates will be cancellative; see the proof of Theorem 4 .

In the finite case, this was done by J. Froemke and the author [3], but the partial groupoids were not cancellative. For the countable case, we could modify their definition slightly, or equivalently, form a prime product of their finite examples. However, the construction of $t(x, y, z)$ in [3] is extremely complicated and tedious to verify. But in the countable case, one has very much more freedom and so it is very much easier to construct $t(x, y, z)$. As we shall see, it is even easy to make the groupoids cancellative.

We start by defining $n \circ n$ to be $n+1$ and $(n+1) \circ n$ to be $n-1$. Thus, for every $k \in Z, f_{k}(n)=n+k$ is a term function. Note that $\circ$ is a well-defined cancellative partial groupoid. As the table for $\circ$ is built up it will be left to the reader to verify that it is well defined and cancellative; this is tedious but straightforward. Let $h_{k}(n)=n \circ f_{k}(n)$. Thus, $h_{k}(n)$ is a term function and represents the $k$ th diagonal; in particular, $h_{0}(n)=f_{1}(n)$ and $h_{-1}(n)=f_{-2}(n)$. Of course, $h_{k}$ is not yet defined for other values of $k$. For $k \in Z$, let $g_{k}(n)=k^{2 n+4}$ for $n \geqslant 0$ and $g_{k}(n)=$ $k^{-2 n+3}$ for $n<0$. Now define $h_{1}(n)$ to be $g_{2}(n)$ and $h_{2}(n)$ to be $g_{6}(n)$. For $m \neq n$ define $h_{1}(n) \circ h_{1}(m)$ to be $g_{10}(n) g_{2}(m)$. Define $\left(h_{1}(n)+1\right) \circ h_{2}(r)$ to be $g_{14}(n) g_{6}(r)$ and $g_{10}(n) g_{2}(m) \circ h_{2}(r)$ to be $g_{10}(n) g_{2}(m) g_{6}(r)$. We are now ready to define the ternary discriminator function. This step again takes place on a diagonal so as to preserve cancellation. Define $h_{3}\left(g_{10}(n) g_{2}(m) g_{6}(r)\right)$ to be $n$ and $h_{3}\left(g_{14}(n) g_{6}(r)\right)$ to be $r$. From this it follows that $h_{3}\left(\left(h_{1}(n) \circ h_{1}(m)\right) \circ h_{2}(r)\right)=t(n, m, r)$ is a term function. Finally, define $h_{-2}\left(h_{2}(n)\right)$ to be 0 ; thus, the constant zero function is a term function and $\circ$ is a well-defined cancellative partial groupoid.

Let $\mathbf{K}$ be the class of all groupoids on $Z$ extending $\circ$ as defined above which are cancellative and such that $m \circ n=1$ only if $m \circ n$ is defined above and equal to 1 . This last condition guarantees that no $A \in \mathbf{K}$ is a quasigroup. Notice that in each row and each column in the table for $\circ$ as defined above there are infinitely many members of $Z$ not occurring; this means that $\mathbf{K}$ contains continuum many members.

TheOrem 4. For every $\underline{A} \in \mathbf{K}, \operatorname{HSP}(\underline{A})$ is equationally complete. $\operatorname{HSP}(\mathbf{K})$ is a discriminator variety of cancellative groupoids such that no nontrivial member is a quasigroup.

Proof. For the first statement, apply Lemma 2. For the second, note that the subdirectly irreducible groupoids in $\operatorname{HSP}(\mathbf{K})$ are the member of $\operatorname{SP}_{u}(\mathbf{K})$. Since cancellativity is preserved by ultraproducts and subalgebras, Lemma 3 implies that each member of $\operatorname{HSP}(\mathbf{K})$ is cancellative. To see that no nontrivial member of $\operatorname{HSP}(\mathbf{K})$ 
is a quasigroup, first note that a quotient of a quasigroup is again a quasigroup. Thus, we need only see that no member of $\operatorname{SP}_{u}(\mathbf{K})$ is a quasigroup. Since the members of $\mathbf{K}$ satisfy $\forall x(-1 \circ x \neq 1)$, so do all members of $\mathrm{SP}_{u}(\mathbf{K})$. Hence, no member of $\mathrm{SP}_{u}(\mathbf{K})$ is a quasigroup.

Problems. (1) It is easy to complete $\circ$ to a quasigroup; this does not imply that the variety generated by the completion is a variety of quasigroups. Is it possible to complete $\circ$ to a quasigroup such that this quasigroup generates a variety of quasigroups?

(2) The techniques of this paper appear to depend essentially on making effective use of the main diagonal (e.g., $n \circ n=n+1$ ). They seem to be totally useless for idempotent groupoids. The following interesting problem remains open: what is the spectrum of a variety of idempotent groupoids?

ACKNOWLEDGEMENT. This paper has benefited greatly from extensive comments from two referees to whom I express my thanks.

\section{REFERENCES}

1. A. D. Bol'bot, Varieties of quasigroups, Sibirsk. Mat. Zh. 13 (1972), 252-271.

2. A. Foster and A. Pixley, Semi-categorical algebras. II, Math. Z. 85 (1964), 169-184.

3. J. Froemke and R. Quackenbush, The spectrum of an equational class of groupoids, Pacific J. Math. 58 (1975), 381-386.

4. B. Jonsson, Algebras whose congruence lattices are distributive, Math. Scand. 21 (1967), 110-121.

5. J. Kalicki, The number of equationally complete classes of equations, Nederl. Akad Wetensch. Proc. Ser. A 58 (1955), 660-662.

6. V. L. Murskii, The existence of a finite basis of identities, and other properties of "almost all" finite algebras, Problemy Kibernet. 30 (1975), 43-56. (Russian)

7. D. Pigozzi, Equational logic and equational theories of algebras, Seminar report, Purdue Univ., Comp. Sci. Dept., 1973.

Department of Mathematics, University of Manitoba, Winnipeg, Manitoba, Canada R3T 2N2 